\section{E-080 GENDER CHARACTERISTICS, COMORBIDITY AND OUTCOMES OF CEREBRAL VENOUS SINUS THROMBOSIS IN A NOVEL U.S. POPULATION}

${ }^{1} S$ Walsh-Blackmore*, ${ }^{2} \mathrm{~J}$ Fraser, ${ }^{3} \mathrm{P}$ Kitzman, ${ }^{3} \mathrm{M}$ Dobbs. ${ }^{1}$ College of Medicine, University of Kentucky, Lexington, $K Y_{;}{ }^{2}$ Neurosurgeny, University of Kentucky College of Medicine, Lexington, KY; ${ }^{3}$ Department of Nuerology, University of Kentucky College of Medicine, Lexington, $K Y$

\subsection{6/neurintsurg-2019-SNIS.155}

Introduction Cerebral Venous Sinus Thrombosis (CVST) has an estimated incidence of $1.32-1.57 / 100,000 / y r$, with death or severe disability in less than $10 \%$. There is a reported elevated incidence in young -middle age females. The majority of reported epidemiologic and outcome data has been collected outside the United States (US). Available US data shows low representation of the Appalachian region, which has relatively a higher burden of disease and lower socioeconomic level. The objective of this study was to examine the gender characteristics of CVST patients in the United States, with greater representation of the Appalachian region.

Methods Data were collected in a retrospective cohort using the Kentucky Appalachian Stroke Registry (KApSR), collected from admissions in a Comprehensive Stroke Center serving 554,300 from the central-eastern United States. All diagnosed CVST patients found in the database from 2010-2018 greater than 18 years of age were included in the data set. Descriptive data were computed using SPSS statistics.

Results 101 patients diagnosed with CVST were included. 58 patients were female $(57.4 \%)$. Median age was 44 years. The National Institutes of Health Stoke Scale (NIHSS) was reported for 32 female and 21 male patients. Median NIHSS was. 5 in females and 0 in males at admission. Median length of stay was 7 days in females vs. 4 days in males. Discharge data were available for 51 female and 33 Male patients. Of female patients, $28(55 \%)$ were discharged to home, 17(33\%) were transferred to continued care, $4(8 \%)$ were transferred to hospice and $2(4 \%)$ died within 48 hours of admission. Of male patients, $25(76 \%)$ were discharged to home, $7(21 \%)$ were transferred to continued care, and 1 (3\%) was transferred to hospice. Female patients had a higher burden or comorbidities and multimorbidity compared to male patients. Conclusion The results of this study indicate a higher burden of comorbidity, more severe presentation, and worse prognosis in female patients. This is in contrast to the majority of CVST research, many of which have found the male sex to be an independent risk factor for worse outcomes. This may reflect a particular variant among the Appalachian population Disclosures S. Walsh-Blackmore: 1; C; University of Kentucky Center for Clnical and Translational Sciences Professional Student Mentored Research Fellowship. J. Fraser: None. P. Kitzman: None. M. Dobbs: None.

\section{E-081 ACUITY CLASSIFICATION SYSTEM FOR NEURO- INTERVENTIONAL SURGERY CASES IN THE EVOLVING WORLD OF HYPERACUTE ENDOVASCULAR STROKE CARE}

${ }^{1} \mathrm{~A}$ Linn, ${ }^{1} Y$ Gujrati, ${ }^{2} \mathrm{O}$ Qahwash, ${ }^{1} \mathrm{~A}$ Razak. 'Dept of Neuro-Intervention, Michigan State University/Sparrow Health System, East Lansing, MI; ${ }^{2}$ Dept of Endovascular Neurosurgery, Lansing Neurosurgery/Sparrow Health System, East Lansing, MI

10.1136/neurintsurg-2019-SNIS.156
Introduction/Purpose NIS (Neuro-Interventional Surgery) as a sub-specialty has undergone a tremendous transformation in many regards since its early beginnings. A major area of change includes the demand for the NIS provider to be immediately available while on call, particularly as it pertains to MER (mechanical endovascular reperfusion) for ELVO (emergency large vessel occlusion) type acute ischemic strokes. MER for ELVO's can be regarded amongst the most pressing hyperacute emergencies in all of medicine, thereby placing a substantial demand on the providers and healthcare facilities offering this treatment. As endovascular stroke care continues to evolve, so too will the number of hyper-emergent NIS cases which have already risen dramatically, leading to an increased potential for NIS provider burnout. We propose an acuity classification system which may assist individual healthcare systems providing NIS care to better assess the degree of change over time and prepare for the future.

Materials and methods We retrospectively reviewed just under 21-months of NIS cases completed at our facility from $7 / 01 /$ 2017 through 3/22/2019. These cases were classified into four categories based upon their level of acuity with regard to how quickly the patient ought to be brought to the neuro-angiography suite for the procedure per the NIS provider's discretion. The type of NIS case deemed 'hyper-emergent' will vary from provider to provider, but by using a classification system based upon the acceptable degree of time delay from the procedure's decision start point to the patient's arrival to the neuro-angiography suite, unique data for an individual healthcare system can be generated and compared over time. In general, hyper-emergent NIS cases include all ELVO's, as well as select cases of intracranial and extracranial hemorrhage.

Results Our healthcare system experienced a notable increase in the number and percentage of hyper-emergent NIS cases relative to less acuity level cases. In our facility, this data will be used for internal healthcare system review for various purposes, including resource allocation and NIS provider on-call coverage assessments. As these data points will most certainly be facility specific, only our facility's current and recent acuity level percentage breakdown is listed for example purposes. For reference, our elective caseload volume has stayed relatively stable over time, confirming a true increase in the hyper-emergent caseload volume by number and percentage.

\begin{tabular}{|c|c|c|c|}
\hline \multirow[t]{2}{*}{$\begin{array}{l}\text { NIS Case } \\
\text { Acuity } \\
\text { Level }\end{array}$} & \multirow[t]{2}{*}{$\begin{array}{l}\text { NIS Decision to Expected } \\
\text { Patient Suite Arrival Time }\end{array}$} & \multicolumn{2}{|l|}{$\begin{array}{l}\text { Our Facility's NIS Case } \\
\text { Percentage Breakdown }\end{array}$} \\
\hline & & $7 / 01 / 2017$ to $6 / 30 / 2018$ & $\begin{array}{l}7 / 01 / 2018 \\
\text { to } 3 / 22 / \\
2019\end{array}$ \\
\hline $\begin{array}{l}\text { A) Hyper- } \\
\text { Emergent }\end{array}$ & Less Than 60 Minutes & $10.6 \%$ & $13.6 \%$ \\
\hline B) Emergent & Less Than 12 Hours & $7.1 \%$ & $12.0 \%$ \\
\hline C) Urgent & Same Hospital Stay & $29.6 \%$ & $28.9 \%$ \\
\hline D) Elective & Outpatient Scheduled Case & $52.7 \%$ & $45.5 \%$ \\
\hline & All Cases & $100.0 \%$ & $100.0 \%$ \\
\hline
\end{tabular}

Conclusion Our healthcare system has observed an increase in the number and percentage of hyper-emergent NIS cases over time, chiefly as a result of the research-driven expansion of the indications and selection of ELVO patients for MER. As 